\title{
Avalanche-ion back-flow reduction in gaseous electron multipliers based on GEM/MHSP
}

\author{
J.M. Maia ${ }^{\mathrm{a}, 1,2}$, D. Mörmann ${ }^{\mathrm{a}}$, A. Breskin ${ }^{\mathrm{a}}$, R. Chechik ${ }^{\mathrm{a}, *}$, J.F.C.A. Veloso ${ }^{\mathrm{b}, \mathrm{c}}$, \\ J.M.F. dos Santos ${ }^{b}$ \\ ${ }^{a}$ Department of Particle Physics, The Weizmann Institute of Science, P.O. Box 26, 76100 Rehovot, Israel \\ ${ }^{\mathrm{b}}$ Physics Department, University of Coimbra, P-3004-516 Coimbra, Portugal \\ ${ }^{\mathrm{c}}$ Physics Department, University of Aveiro, P-3810-193 Aveiro, Portugal
}

Received 5 August 2003; received in revised form 24 November 2003; accepted 1 December 2003

\begin{abstract}
We report on avalanche-ion back-flow measurements in the novel Micro-Hole and Strip-Plate (MHSP) multiplier and in gaseous photomultipliers comprising Gas Electron Multipliers (GEMs) followed by an MHSP. In a 3-GEMs/ MHSP photomultiplier with reflective photocathode, avalanche-ion back-flow fraction of $\sim 7 \%$ and $\sim 2 \%$ were recorded for respective effective gains of $10^{7}$ and $10^{6}$, in $\mathrm{Ar} / \mathrm{CH}_{4}(95 / 5)$ at 760 Torr. This is about one order of magnitude reduction in ion back-flow compared to the best values measured in 4-GEMs photomultiplier at the same gain. We describe the mode of operation of the MHSP and explain its ion back-flow reduction features.
\end{abstract}

(C) 2004 Elsevier B.V. All rights reserved.

PACS: $29.40 .-\mathrm{n} ; 29.40 . \mathrm{Cs} ; 85.60 . \mathrm{Gz}$

Keywords: Gas avalanche electron multipliers; Gaseous photomultipliers; Ion back-flow; GEM; MHSP

\section{Introduction}

\subsection{Motivation}

The impact of avalanche-ions on the photocathode limits the performance of gaseous photo-

\footnotetext{
*Corresponding author. Tel.: +972-8-934-2645; fax: +9728-934-2611.

E-mail addresses: jmaia@gian.fis.uc.pt (J.M. Maia), rachel. chechik@weizmann.ac.il (R. Chechik).

${ }^{1}$ Also corresponding author. Tel.: + +351-239-410667; fax: $++351-239-829158$

${ }^{2}$ Presently at University of Coimbra and University of BeiraInterior, Covilhã, Portugal.
}

multipliers [1] for the UV and visible spectral ranges. Ions impact affects the quantum efficiency (QE) of the photocathode [2] by continuous modifications of its structure and by electrostatic charging of the photocathode surfaces, which lead to electric field-lines modification. Even more serious is the ion-induced secondary electron emission from the photocathode, resulting in divergence from the Townsend-avalanche multiplication process and leading to instabilities and breakdown.

In recent years there has been considerable progress in gaseous photomultipliers (GPMTs) combining a cascade of Gas Electron Multiplier 
(GEMs) [3] and semi-transparent [4] or reflective photocathodes [5]. In such detectors, the avalanche-ion back-flow follows the electric field lines and it has been shown [6] that reducing the electric field at the photocathode surface could reduce the ion-impact on the photocathode. However, this approach is not always acceptable; an efficient detection of single photons requires the highest possible QE, which in gaseous photomultipliers imposes high (typically $>1 \mathrm{kV} / \mathrm{cm}$ ) electric fields at the photocathode surface. Unfortunately, under high electric fields, that reduce photoelectrons backscattering [7], the ions are more energetic, resulting in more damage to the photocathode.

Reducing the avalanche-ion back-flow, i.e. the fraction of avalanche-ions that can hit the photocathode, in GEM-based photomultipliers is an important and difficult task, extensively studied recently [6-9]. Similar studies, relevant to GEMbased tracking detectors and TPCs have also been carried out $[10,11]$. Some reduction in ion backflow could be achieved by optimizing electric-field ratios along the GEM cascade, or by varying geometrically the GEM transparency [8]. The ioninduced secondary electron-emission, namely the positive ion-feedback, strongly depends upon the photocathode material. Most sensitive are visible spectral-range photocathodes, having low emission threshold due to their small band gap and low electron affinity. It has been recently demonstrated that the ion-feedback in multi-GEM GPMTs with bi-alkali photocathodes, depends on the gas composition [12]. This dependence is derived from the differences in the ionization energy of the ions involved, as well as from the differences in electron backscattering and in ion back-flow.

In this work we propose to reduce the ion backflow fraction, using some particular properties of the recently introduced gas electron multiplier, the Micro-Hole and Strip-Plate (MHSP) [13-16]. The MHSP combines the characteristics of both the GEM and the microstrip gas chamber (MSGC) [17] in a single, double-sided insulating plate. It consists of two independent charge-amplification stages, micro-patterned on a single thin Kapton substrate, metalized on both faces (Fig. 1); it comprises GEM-like holes on the front face, and MSGC-like anode- and cathode-strips on the rear metalized face. A potential difference between the top face and the cathode-strips and between the latter and the anode-strips, establish a dipolar electric field within the holes, and a strong electric field near the thin anode-strips. Electrons originating from the gas volume above the MHSP (Fig. 2) are focused into the holes, whereby an avalanche occurs under the high electric field; the avalanche electrons are further multiplied on the anodestrips, where the final charge is collected. Ions moving away of the anode-strips induce charge on the neighbouring cathode-strips and cathode-
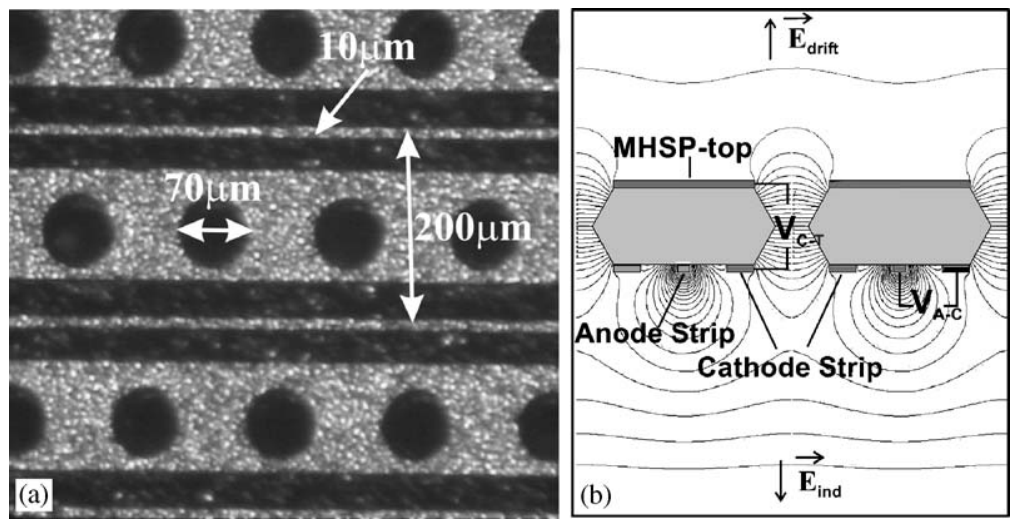

Fig. 1. Photomicrograph of the stripped-side (MS) of an MHSP electron multiplier (a), and schematic view of MHSP equipotentials lines (b) for $V_{\mathrm{A}-\mathrm{C}}=300 \mathrm{~V}, V_{\mathrm{C}-\mathrm{T}}=400 \mathrm{~V}, E_{\text {ind }}=-6.0 \mathrm{kV} / \mathrm{cm}$ and $E_{\mathrm{drift}}=1.0 \mathrm{kV} / \mathrm{cm}$. The reverse induction field is used for capturing part of the anode-strips avalanche ions on a cathode-plane localized below the MHSP. 


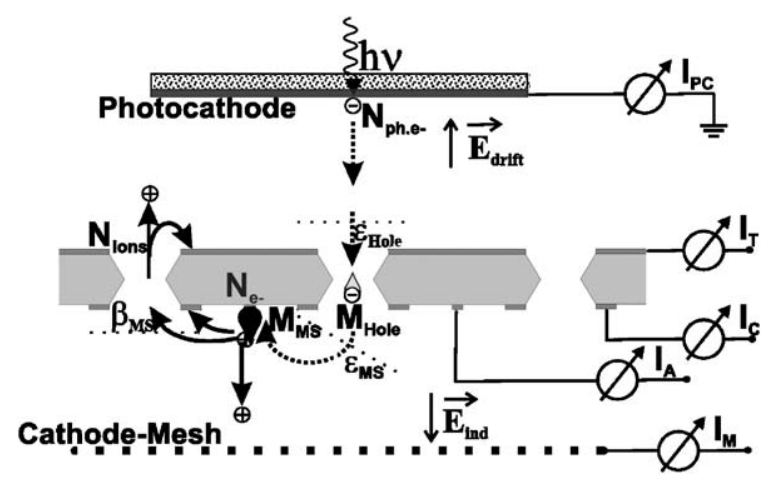

Fig. 2. Schematics of the single MHSP detector coupled to a semi-transparent photocathode. The notations and the scheme of electrons/ions transport parameters are also shown.

plane/mesh (Fig. 2). A single MHSP-electrode can provide total effective gain exceeding $10^{3}$, similar to a GEM $[15,16]$.

The geometry of the MHSP, with the final avalanche totally optically screened from the photocathode by the multiplier's substrate, and where a substantial part of the ions can be trapped on the cathode -strips and -plane, may present an interesting solution for ion- and photon- feedback suppression in gas avalanche electron multipliers.

In the present work, we demonstrate the capability of an MHSP in reducing the ion backflowing; we report on the ion back-flow suppression in a GPMT comprising 3-GEMs with a reflective CsI-photocathode deposited on the topplane of the first GEM, and with an MHSP as a last multiplication stage. We discuss the ion collection mechanism responsible for the $\sim 10$ fold reduction in ion back-flow compared to a 4GEMs gaseous photomultiplier (GPMT).

\subsection{Theoretical considerations}

Using an MHSP, there are different ways to neutralize the strips-avalanche ions, as shown in Fig. 2: (i) on nearby cathode-strips; (ii) on any cathode-plane/mesh localized below the MHSP; and (iii) on MHSP-top electrode. The special feature of the MHSP is that the majority of avalanche ions produced at the anode-strips are collected on the nearby cathode-strips, but in addition we can explore the possibility of capturing part of the avalanche ions on the cathodeplane below the MHSP, by applying a moderate reverse electric field, $E_{\text {ind }}$. The ions back-flowing into the MHSP holes and the ions produced in the first avalanche within the holes are collected on the MHSP-top or on the photocathode, depending on the ratio between the drift field $E_{\text {drift }}$, and the holefield (defined by $V_{\mathrm{C}-\mathrm{T}}$ ).

\subsubsection{Single MHSP multiplier}

To evaluate the performance of the MHSP regarding the positive ions back-flow, we defined the parameter $\varphi$ that measures the fraction of ions created at the MHSP (hole and anode-strips) and propagating backward through the holes.

For an MHSP electron multiplier shown in Fig. 2 the fraction $\varphi$ of ions back-flowing is given by

$$
\begin{aligned}
\varphi & =\frac{N_{\text {ions }}}{N_{\text {e- }}} \\
& =\frac{N_{\text {ph.e- }} \varepsilon_{\text {Hole }}\left[\beta_{\mathrm{MS}} \varepsilon_{\mathrm{MS}} M_{\mathrm{MS}} M_{\mathrm{Hole}}+M_{\mathrm{Hole}}\right]}{N_{\text {ph.e- }} \varepsilon_{\mathrm{Hole}} \varepsilon_{\mathrm{MS}} M_{\mathrm{Hole}} M_{\mathrm{MS}}} \\
& =\beta_{\mathrm{MS}}+\frac{1}{\varepsilon_{\mathrm{MS}} M_{\mathrm{MS}}}
\end{aligned}
$$

where $N_{\text {ions }}$ is the total number of ions that leave the holes; $N_{\mathrm{e}}$ is the total number of avalancheelectrons that are collected on the anode-strips and is approximately equal to the number of anodestrips avalanche ions for high anode-strips gains; $\beta_{\mathrm{MS}}$ is the fraction of ions produced in the anodestrips avalanche entering the holes (i.e. the iontransparency of the micro-strip (MS) electrode); $N_{\text {ph.e- }}$ is the number of photoelectrons emitted by the photocathode; $\varepsilon_{\text {Hole }}$ is the photo-electron collection efficiency into the holes; $M_{\mathrm{MS}}$ and $M_{\text {Hole }}$ are the real gains at the anode-strips and in the MHSP-holes, respectively, and $\varepsilon_{\mathrm{MS}}$ is the electron extraction efficiency from the holes to the anode-strips. The two terms in Eq. (1) represent the contributions from the anode-strips avalanche ions and the hole-avalanche ions, respectively. For large anode-strips gains the first term is dominant. Indeed, the anode-strips effective gain is given by $G_{\mathrm{MS}}=\varepsilon_{\mathrm{MS}} M_{\mathrm{MS}}$; consequently we may write

$\varphi=\beta_{\mathrm{MS}}+\frac{1}{G_{\mathrm{MS}}}$ 
Eqs. (1) and (2) are valid only if $M_{\mathrm{MS}}$ and $M_{\mathrm{Hole}}$ are $\gg 1$. The two parameters of Eq. (2) depend not only on the geometry of the MHSP electrode and on the gas mixture and pressure, but also on the electric fields in the adjacent regions of the MHSP. The ion-transparency $\beta_{\mathrm{MS}}$ of the MS-electrode is determined by the hole-field (defined by $V_{\mathrm{C}-\mathrm{T}}$ ), the strips-field (defined by $V_{\mathrm{A}-\mathrm{C}}$ ), and the induction field $E_{\text {ind }}$ (Figs. 1 and 2). The MS effective gain, $G_{\mathrm{MS}}$, is determined essentially by the voltages $V_{\mathrm{A}-\mathrm{C}}$ and $V_{\mathrm{C}-\mathrm{T}}$.

The ion-transparency of the MHSP, $\beta_{\mathrm{MHSP}}$, is defined as the fraction of ions produced in the anode-strips avalanche that enter, and then leave the MHSP-holes. This parameter is equal to the product of the MS ion-transparency, $\beta_{\mathrm{MS}}$, and the parameter $\beta_{\text {top }}$, which is the number of ions that leave the MHSP-holes normalized to the number of ions that enter the holes:

$\beta_{\mathrm{MHSP}}=\beta_{\mathrm{MS}} \beta_{\text {top }}$

\subsubsection{Multi-GEM/MHSP multiplier}

Concerning the ion back-flow in a GPMT, it is useful to define $\xi$, the fraction of avalanche-ions back-flowing into the photocathode, i.e. the ratio between the number of ions neutralized on the photocathode and the number of electrons collected at the MS-anode. Evaluating $\xi$ in a multiGEM/MHSP GPMT requires good knowledge of the multiplier's geometry (e.g. hole-diameter, holeshape, etc.), the electric fields, the gas mixture and pressure, etc. The dependence on the gas mixture and pressure in multi-GEM GPMTs was already discussed in Ref. [8], and it was shown that $\xi$ is practically independent of these two parameters, and that the most important factors are the multiplier's geometry and the electric fields.

For the 3-GEM/MHSP/reflective-photocathode GPMT, shown in Fig. 3, and using considerations similar to Eq. (1), we can express $\xi$ as

$$
\begin{aligned}
\xi= & \frac{N_{\text {ions }}^{\mathrm{PC}}}{N_{\mathrm{e}-}}=\beta_{\mathrm{MS}} \beta_{3} \beta_{2} \beta_{1}+\frac{\beta_{3} \beta_{2} \beta_{1}}{\varepsilon_{\mathrm{MS}} M_{\mathrm{MS}}}+\frac{\beta_{2} \beta_{1}}{\varepsilon_{\mathrm{MS}} M_{\mathrm{MS}} \varepsilon_{3} M_{4}} \\
& +\frac{\beta_{1}}{\varepsilon_{\mathrm{MS}} M_{\mathrm{MS}} \varepsilon_{3} M_{4} \varepsilon_{2} M_{3}} \\
& +\frac{1}{\varepsilon_{\mathrm{MS}} M_{\mathrm{MS}} \varepsilon_{3} M_{4} \varepsilon_{2} M_{3} \varepsilon_{1} M_{2}}
\end{aligned}
$$

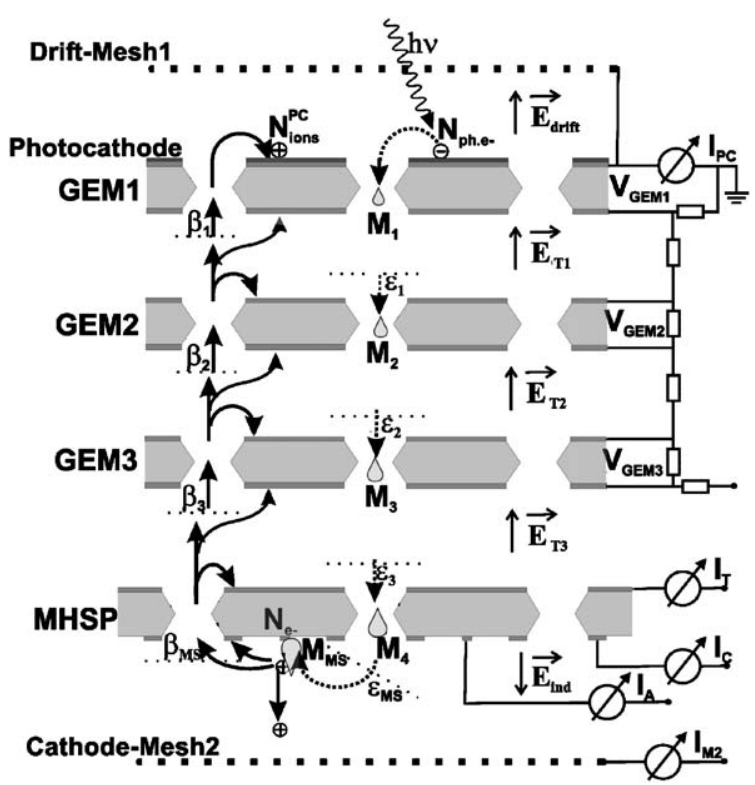

Fig. 3. Schematics of the multi-GEM/MHSP detector with a reflective photocathode deposited on the GEM1-top. The notations and the scheme of electrons/ions transport parameters are also shown.

where $N_{\text {ions }}^{\mathrm{PC}}$ is the number of back-flowing ions collected at the photocathode; $N_{\mathrm{e}-}, \beta_{\mathrm{MS}}, \varepsilon_{\mathrm{MS}}$ and $M_{\mathrm{MS}}$ are defined as above; $\beta_{i}(i=1,2,3)$ is the ratio between the number of ions entering the holes of structure $i$ and those leaving the holes of the structure $i+1, M_{i}(i=1,2,3,4)$ is the real gain in the holes of structure $i$ and $\varepsilon_{i}$ is the electron extraction/collection efficiency from the holes of structure $i$ to the holes of structure $i+1$.

The first term in Eq. (4) represents the contribution from ions produced at the anode-strips and it involves the ion-transparency of the whole multiplier cascade. The following terms represent the contributions from ions produced in the respective cascade elements, namely from the MHSP-holes and the preceding GEMs-holes, and become negligible if a high effective gain is obtained at the anode-strips.

\section{Experimental set-up and methodology}

The photocathode and the micro-pattern electron multipliers were mounted inside a stainlesssteel chamber filled at a gas pressure of 760 Torr. 
An $\mathrm{Ar} / \mathrm{CH}_{4}(95 / 5)$ gas mixture was used, in flow mode, for its good proprieties of gain and photoelectron extraction efficiency from the photocathode [4]. The photocathode was illuminated with an $\mathrm{Hg}(\mathrm{Ar}) \mathrm{DC}$ UV-lamp, and attenuators were used to maintain the currents on the various electrodes below charging-up levels.

The GEMs and MHSPs ${ }^{3}$ are produced from $50 \mu \mathrm{m}$ Kapton and $5 \mu \mathrm{m}$ copper clad on both sides, and have an active area of $2.8 \times 2.8 \mathrm{~cm}^{2}$. The MHSP (Fig. 1) has bi-conical holes of diameter $50 \mu \mathrm{m}$ in the Kapton and $70 \mu \mathrm{m}$ in the copper, arranged in a hexagonal lattice, with two edges of $140 \mu \mathrm{m}$ and four edges of $210 \mu \mathrm{m}$; the optical transparency is $\cong 7 \%$. The MHSP micro-strip pattern has a $200 \mu \mathrm{m}$ pitch with anodes and cathodes widths of 10 and $120 \mu \mathrm{m}$, respectively. The anode-cathode gap is $35 \mu \mathrm{m}$. The GEMs have a hexagonal layout, of $140 \mu \mathrm{m}$ edges, with biconical holes of 50 and $70 \mu \mathrm{m}$ in the Kapton and copper, respectively; with an optical transparency $\cong 12 \%$. The meshes are made of stainless-steel woven crossed wires, $50 \mu \mathrm{m}$ diameter and $500 \mu \mathrm{m}$ pitch, with optical transparency $\cong 81 \%$.

The currents on the different electrodes of the photodetector were recorded with four-floating Fluke 175 True RMS $10 \mathrm{M} \Omega$ input impedance digital multimeters and a grounded analogue electrometer Keithley 610C. The digital multimeters provided the currents by recording the voltage drop on a $33 \mathrm{M} \Omega$ resistor, placed between the power-supply and the electrode. The accuracy of the measurements was verified against the more precise analogue electrometer, under the same electrostatic conditions, and it was found to be adequate for currents $\geqslant 0.5 \mathrm{nA}$.

The effective gain $G$, of the photodetector is determined by the electron current collected on the anode $I_{\mathrm{A}}$, normalized to the photoelectron current, $I_{\text {ph.e }}$, of the electrons emitted from the photocathode in extraction mode; $G=I_{\mathrm{A}} / I_{\text {ph.e }}$.

In single MHSP multiplier studies, a $300 \AA$ thick semi-transparent CsI-photocathode was evaporated on a $2.5 \mathrm{~mm}$ thick quartz window, preevaporated with $100 \AA$ thick $\mathrm{Al}$ to provide the

\footnotetext{
${ }^{3}$ The GEMs and MHSPs are produced at the Printed Circuits Workshop, CERN, Geneva.
}

electric contact. The MHSP was placed $4 \mathrm{~mm}$ below the photocathode (PC), and $3 \mathrm{~mm}$ above a stainless-steel cathode-mesh (M) (Fig. 2). During this study, the drift field $E_{\text {drift }}$, was kept constant at $0.5 \mathrm{kV} / \mathrm{cm}$, providing $\cong 90 \%$ of the maximum photoelectron current from the photocathode. The photocathode was connected to a grounded electrometer, and the other 4-electrodes of the photodetector were individually connected to HVpower supplies and to the digital multimeters, as explained above. This arrangement provides data on the distribution of all the charges, electrons and ions, under various operation conditions.

Under all circumstances the sum of all measured currents should be zero:

$I_{\mathrm{PC}}+I_{\mathrm{T}}+I_{\mathrm{C}}+I_{\mathrm{A}}+I_{\mathrm{M}}=0$

where $I_{\mathrm{PC}}, I_{\mathrm{T}}, I_{\mathrm{C}}$, and $I_{\mathrm{M}}$ are the currents measured on the photocathode, MHSP-top, cathode-strips and mesh, respectively.

At high anode-strips gain the fraction $\varphi$ of ions created at the MHSP (holes and anode-strips) and collected above the MHSP-holes, can be derived from the ion currents on the MHSP-top, $I_{\mathrm{T}}$, and on the photocathode, $I_{\mathrm{PC}}$, and the electron current on the anode-strips, $I_{\mathrm{A}}$ :

$\varphi=\frac{I_{\mathrm{PC}}+I_{\mathrm{T}}}{I_{\mathrm{A}}}$

In multi-GEM/MHSP multiplier studies, a cascade of 3-GEMs and a MHSP were mounted between two meshes $\left(M_{1}\right.$ and $\left.M_{2}\right)$, as shown in Fig. 3. A $2500 \AA$ thick layer of reflective CsIphotocathode was evaporated on the top-side of the Au-plated first GEM. The distances between successive elements were as follows: $1.5 \mathrm{~mm}$ between GEMs; $2 \mathrm{~mm}$ between the third GEM and the MHSP; $3 \mathrm{~mm}$ between the MHSP and $\mathrm{M}_{2}$, and between the first GEM and $M_{1}$.

The photocathode and the mesh $\mathrm{M}_{1}$ were interconnected to guarantee an optimal condition for photoelectron extraction in the reflective photocathode geometry, i.e. $E_{\mathrm{drift}} \cong 0$ [5], and were connected to a grounded electrometer. The others 5-GEM electrodes were polarized by a voltagedivider connected to a single HV-power supply. The voltage difference across the first GEM was maintained at $\sim 350 \mathrm{~V}$, to guarantee a sufficiently 
high electric field on the photocathode surface, for an efficient extraction of photoelectrons and their focusing into the GEM-holes [5]. The MHSPelectrodes and the mesh $\mathbf{M}_{2}$, were connected separately to HV- power supplies, and to the digital multimeters, as above.

The parameter $\xi$, i.e. the fraction of avalancheions back-flowing into the photocathode, is derived from the ratio between the photocathode ion-current $I_{\mathrm{PC}}$, and the electron-current, $I_{\mathrm{A}}$, collected on the anode-strips:

$\xi=I_{\mathrm{PC}} / I_{\mathrm{A}}$

$I_{\mathrm{PC}}$ actually equals the total current recorded from the photocathode under detector multiplication, since the photoelectron-current $I_{\mathrm{ph} . \mathrm{e}^{-}}$is negligible in this case.

\section{Results and discussion}

\subsection{Single MHSP multiplier}

Fig. 4 depicts the gain $G_{\text {MHSP }}$ as function of $V_{\mathrm{A}-\mathrm{C}}$, for a single MHSP-based photodetector (Fig. 2). A total gain $G_{\text {MHSP }} \sim 7 \times 10^{3}$ with anode-

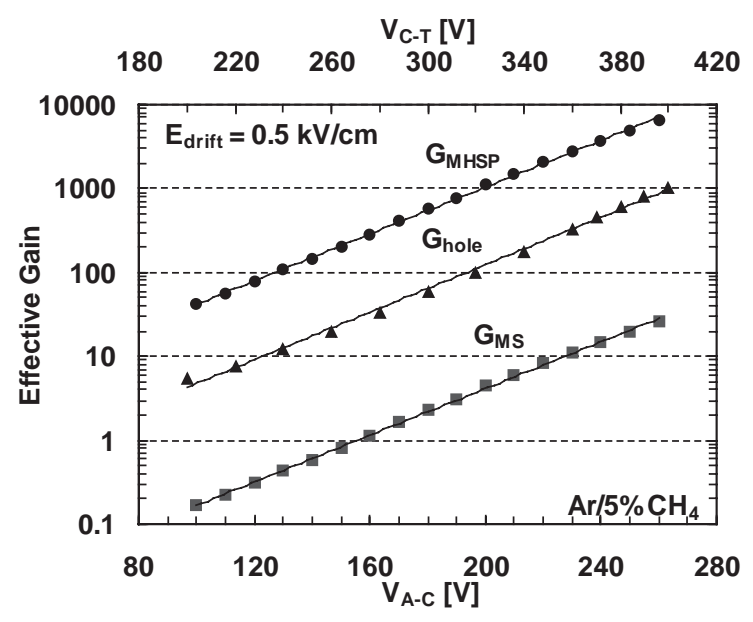

Fig. 4. Gain curves of single MHSP detector: $(\bullet)$ the MHSP gain, $G_{\mathrm{MHSP}}$, and ( $\left.\boldsymbol{\square}\right)$ the anode-strips gain, $G_{\mathrm{MS}}$, are given as a function of the strips-voltage, $V_{\mathrm{A}-\mathrm{C}}$, with $V_{\mathrm{C}-\mathrm{T}}=350 \mathrm{~V}$ and $E_{\text {ind }}=-5.0 \mathrm{kV} / \mathrm{cm} ;(\boldsymbol{\Delta})$ the hole gain, $G_{\text {hole }}$, is given as function of the hole-voltage, $V_{\mathrm{C}-\mathrm{T}}$, with $E_{\text {ind }}=-0.2 \mathrm{kV} / \mathrm{cm}$ for the MHSP operating in a "GEM-mode". strips gain $G_{\mathrm{MS}} \sim 30$, were obtained. The anodestrips gain is estimated from the knowledge of the hole gain, which was measured separately with the MHSP in GEM-mode, i.e. with the anode and cathode strips interconnected and with a small reversed induction field $E_{\text {ind }}=-0.2 \mathrm{kV} / \mathrm{cm}$, to ensure that all electrons are collected on anodecathode strips.

Fig. 5 depicts the dependence of $\varphi$ on $V_{\mathrm{A}-\mathrm{C}}$ for two values of the induction field: (i) $E_{\text {ind }}=-0.2 \mathrm{kV} / \mathrm{cm}$; (ii) $E_{\text {ind }}=-5.0 \mathrm{kV} / \mathrm{cm}$, and for a fixed voltage across the holes, $V_{\mathrm{C}-\mathrm{T}}=200 \mathrm{~V}$. Increasing the strips-voltage $V_{\mathrm{A}-\mathrm{C}}$, results in a decrease of $\varphi$, due to the increase of ions fraction collected on the cathode-strips, and to the decrease of ions fraction produced inside the holes. The curves reach asymptotic values of about 0.4 and 0.23 for the respective $E_{\text {ind }}$ values. At $E_{\text {ind }}=-0.2 \mathrm{kV} / \mathrm{cm}$, a significant fraction of the avalanche-ions created at the anode-strips are collected on the nearby cathode-strips. At $-5.0 \mathrm{kV} / \mathrm{cm}$, in addition, a fraction of these ions are deviated and collected on the cathode-mesh.

For comparison, Fig. 5 also shows $\varphi$ for a MHSP operating in a GEM-mode. As expected, in this case $\varphi \cong 1.0$, i.e. all the ions produced in the

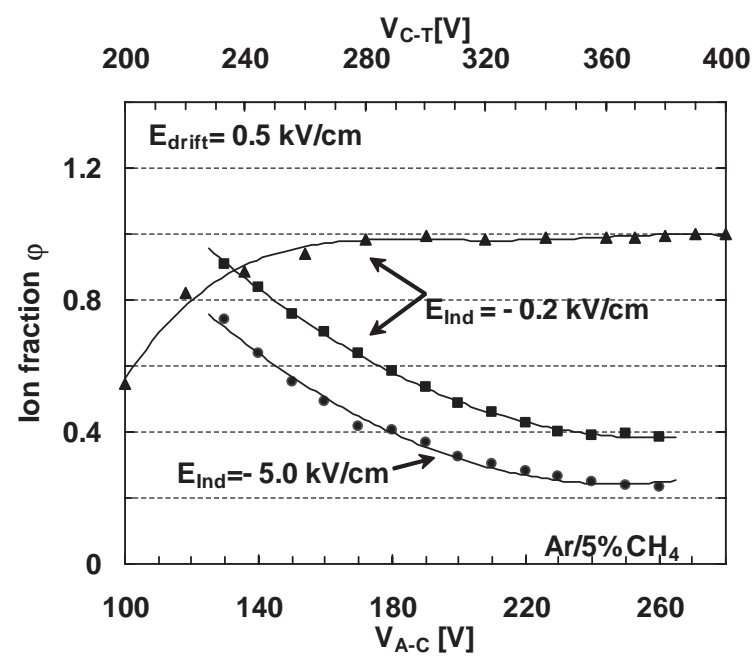

Fig. 5. The fraction $\varphi$ of ions back-flowing in the MHSP-holes, versus the strips-voltage, $V_{\mathrm{A}-\mathrm{C}}$, for $V_{\mathrm{C}-\mathrm{T}}=200 \mathrm{~V}$ : $E_{\text {ind }}=-0.2 \mathrm{kV} / \mathrm{cm}$ and $(\bullet) E_{\text {ind }}=-5.0 \mathrm{kV} / \mathrm{cm}$. For comparison, $\varphi$ versus the hole-voltage, $V_{\mathrm{C}-\mathrm{T}}$, with $E_{\text {ind }}=-0.2 \mathrm{kV} / \mathrm{cm}$ is also shown for the MHSP operating in a "GEM-mode" ( $\boldsymbol{\Lambda})$. 
holes are flowing backwards and collected on the MHSP-top and on the photocathode. For $V_{\mathrm{C}-\mathrm{T}}<300 \mathrm{~V}$, the deviation from $\varphi \cong 1.0$, can be explained by the lost of electrons to the MHSP-top electrode, that is not negligible relatively to the ions collected on it (see Eq. (6)).

The reversed induction field $E_{\text {ind }}$, has an important role in reducing the ion transparency of the MS-electrode, $\beta_{\mathrm{MS}}$. $\beta_{\mathrm{MS}}$ (derived from Eq. (2)), the effective gain $G_{\text {MHSP }}$ and the effective anode-strips gain $G_{\mathrm{MS}}$, are plotted in Fig. 6 as function of $E_{\text {ind }}$. For increasing negative $E_{\text {ind }}$ values, $\beta_{\mathrm{MS}}$ decreases due to the sharing of the ions between the cathode-strips and the cathodemesh; for increasing positive $E_{\text {ind }}$ values, $\beta_{\mathrm{MS}}$ also decreases as more ions are collected on the cathode-strips. In both cases there seems to be a saturation of these effects, and the value of $\beta_{\mathrm{MS}}$ stabilizes at $\sim 0.2$ and 0.17 for positive and negative $E_{\text {ind }}$ values, respectively. In the negative range, $E_{\text {ind }}$ has no significant effect on the effective gains $G_{\mathrm{MHSP}}$ and $G_{\mathrm{MS}}$, while in the positive range we can observe a decrease of the gains, of up to $70 \%$.

Fig. 7 shows the dependence of $\varphi$ on the effective gain $G_{\mathrm{MHSP}}$ measured by varying the voltage $V_{\mathrm{A}-\mathrm{C}}$, for various values of the holevoltage $V_{\mathrm{C}-\mathrm{T}}$. All the curves show a steep drop with the effective gain $G_{\mathrm{MHSP}}$ (i.e. with $V_{\mathrm{A}-\mathrm{C}}$ ) and an asymptotic leveling of $\varphi$. The asymptotic $\varphi$

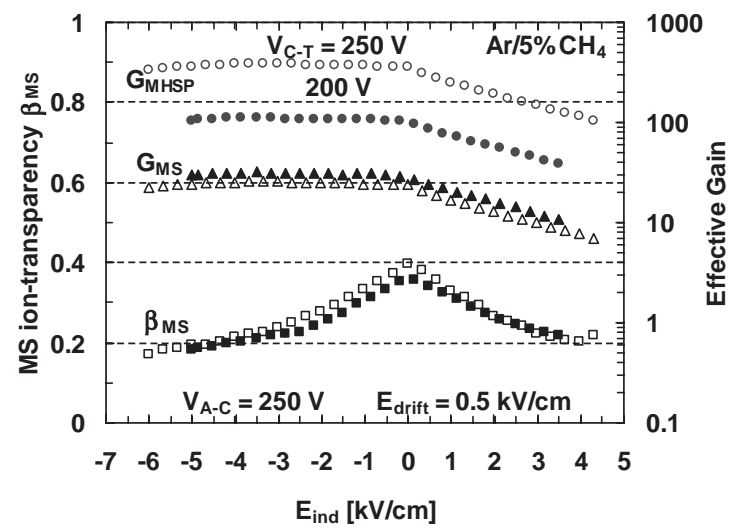

Fig. 6. MS ion-transparency, $\beta_{\mathrm{MS}}(\boldsymbol{\square}, \square)$; MHSP gain, $G_{\mathrm{MHSP}}$ $(\bullet, \bigcirc)$; and anode-strips gain, $G_{\mathrm{MS}}(\boldsymbol{\Lambda}, \triangle)$, versus the induction field, $E_{\text {ind }}$, for hole-voltage values: $V_{\mathrm{C}-\mathrm{T}}=200 \mathrm{~V}$ (full symbols); $V_{\mathrm{C}-\mathrm{T}}=250 \mathrm{~V}$ (open symbols).

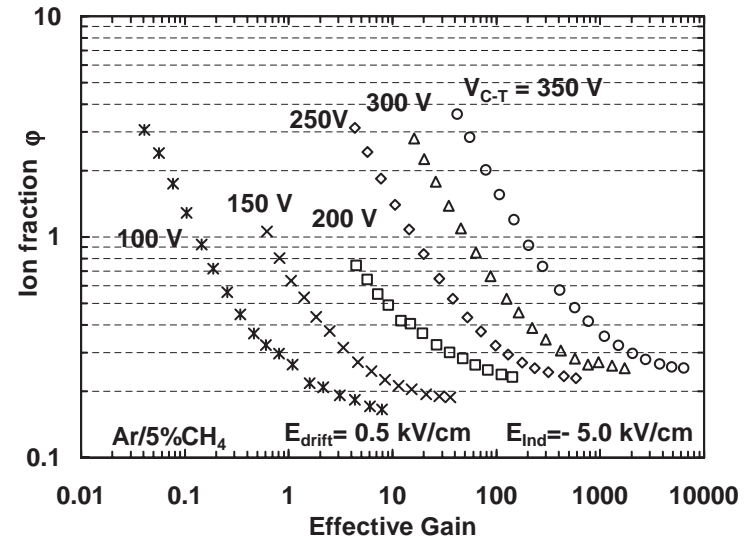

Fig. 7. The fraction $\varphi$ of ions propagating upwards in the holes, as a function of effective gain $G_{\mathrm{MHSP}}$ (measured by varying the strips-voltage $V_{\mathrm{A}-\mathrm{C}}$ ) for several values of holevoltage $V_{\mathrm{C}-\mathrm{T}}$.

values, determined at $V_{\mathrm{A}-\mathrm{C}}=260 \mathrm{~V}$, vary between $\sim 0.17$ and 0.25 , while $G_{\text {MHSP }}$ spans over three orders of magnitude (from $\sim 8$ to $7 \times 10^{3}$ ). The corresponding $G_{\mathrm{MS}}$ values at $V_{\mathrm{A}-\mathrm{C}}=260 \mathrm{~V}$ vary from $\sim 45$ to 25 .

The corresponding ion-transparency of the MSelectrode $\beta_{\mathrm{MS}}$ determined from Eq. (2), varies between $\sim 0.15$ and 0.22 . The asymptotic behaviour of $\varphi$ seen in all the curves at high $V_{\mathrm{A}-\mathrm{C}}$ values, implies that a further increase in the stripsvoltage will not significantly reduce $\varphi$. Increasing $G_{\mathrm{MS}}, \varphi$ may be further reduced by $10-15 \%$ at most, reaching at best the value of $\beta_{\mathrm{MS}}$ (Eq. (2)).

From the studies above we can conclude that the hole-voltage $V_{\mathrm{C}-\mathrm{T}}$ has only a small influence on $\varphi$. More effective are the strips voltage $V_{\mathrm{A}-\mathrm{C}}$ and the induction field $E_{\text {ind. }}$. The best results for $\varphi$ are obtained for the maximum attainable values of strips-voltage, i.e. around $260 \mathrm{~V}$, and at values of induction field in the range of -5.0 to $-6.0 \mathrm{kV} / \mathrm{cm}$. For the above conditions, the MHSP allows a 5fold suppression of ion back-flow relatively to a single GEM.

\subsection{Multi-GEM/MHSP multiplier}

The effective gain $G_{\text {total }}$ of the 3-GEMs/MHSP multiplier as function of $V_{\mathrm{A}-\mathrm{C}}$, is reported in Fig. 8. Very high gains, in excess of $10^{7}$, were 


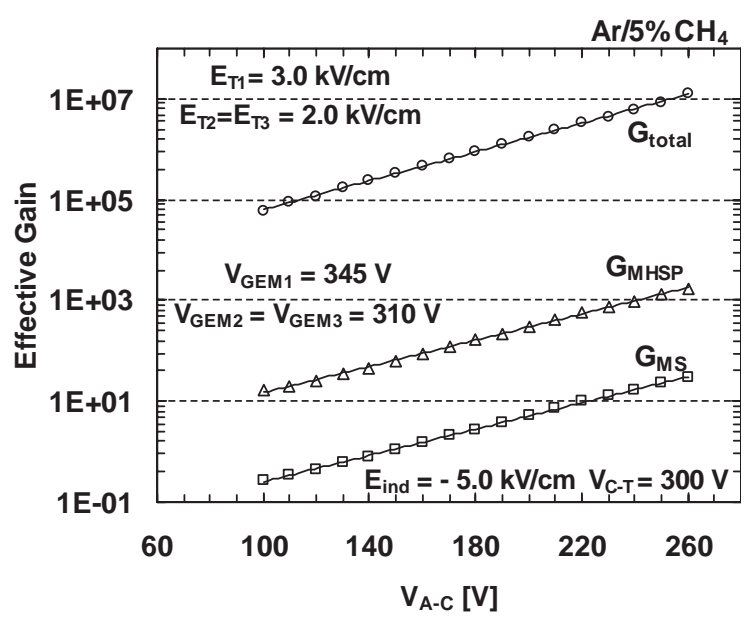

Fig. 8. Gain curves in the multi-GEM/MHSP detector as a function of the strips-voltage, $V_{\mathrm{A}-\mathrm{C}}$, with $V_{\mathrm{C}-\mathrm{T}}=300 \mathrm{~V}$ : $(\bigcirc)$ the total gain, $G_{\text {total }} ;(\triangle)$ the MHSP gain, $G_{\mathrm{MHSP}}$; and $(\square)$ the anode-strips gain, $G_{\mathrm{MS}}$.

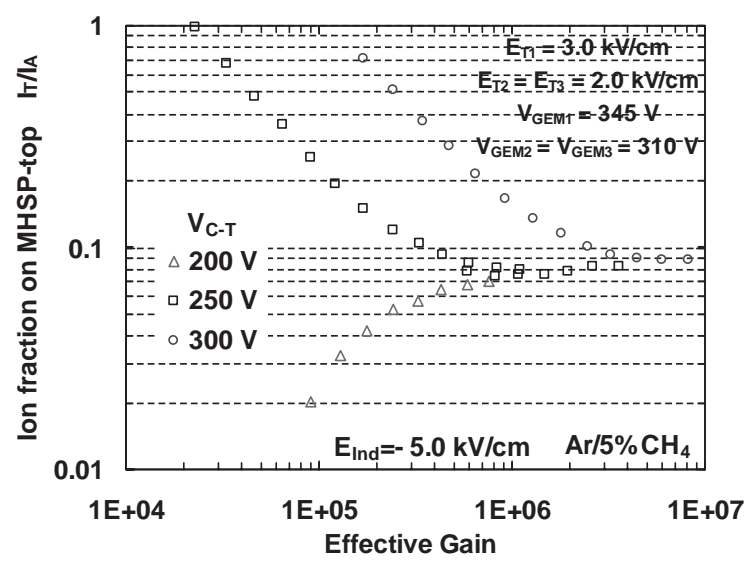

Fig. 9. The fraction of ions collected at the MHSP-top electrode, $I_{\mathrm{T}} / I_{\mathrm{A}}$, as function of the effective gain $G_{\text {total }}$ (measured by varying $V_{\mathrm{A}-\mathrm{C}}$ ) for different $V_{\mathrm{C}-\mathrm{T}}$ values, in the multi-GEM/MHSP detector.

obtained at $V_{\mathrm{C}-\mathrm{T}}=300 \mathrm{~V}$. The corresponding effective gains $G_{\mathrm{MHSP}}$ and $G_{\mathrm{MS}}$ are also shown.

Fig. 9 presents $I_{\mathrm{T}} / I_{\mathrm{A}}$, the fraction of ions collected at the MHSP-top electrode, as function of the effective gain $G_{\text {total }}$, measured by varying $V_{\mathrm{A}-\mathrm{C}}$, for different $V_{\mathrm{C}-\mathrm{T}}$ values. For $V_{\mathrm{C}-\mathrm{T}}=200 \mathrm{~V}$, the $I_{\mathrm{T}} / I_{\mathrm{A}}$ curve deviates from the other curves, of $V_{\mathrm{C}-\mathrm{T}}=250 \mathrm{~V}$ and $V_{\mathrm{C}-\mathrm{T}}=300 \mathrm{~V}$, due to the loss of electrons to the MHSP-top electrode, which is not negligible relative to the ions collected on it. At high gains, the ratio $I_{\mathrm{T}} / I_{\mathrm{A}}$ converges to an asymptotic value of about $0.07-$ 0.09 , dependent slightly on $V_{\mathrm{C}-\mathrm{T}}$. For $V_{\mathrm{C}-\mathrm{T}}=250 \mathrm{~V}$, we have $I_{\mathrm{T}} / I_{\mathrm{A}}=0.08$. Using Eq. (2) and from Figs. $6-7$ one gets $\varphi \cong 0.23$ and $\beta_{\mathrm{MS}} \cong 0.21$. From Eq. (3), and considering $\beta_{\text {top }} \approx 1-\left(I_{\mathrm{T}} / I_{\mathrm{A}}\right) / \varphi=0.65$, one gets a value for the MHSP ion-transparency $\beta_{\mathrm{MHSP}}=0.21 \times$ $0.65 \cong 0.14$. This is the fraction of ions produced at anode-strips and flowing to stages above the MHSP. Thus, the fraction of ions neutralized on the various MHSP electrodes is about $86 \%$.

In Fig. 10 we present $\xi$ as function of the strips-voltage $V_{\mathrm{A}-\mathrm{C}}$ for three $V_{\mathrm{C}-\mathrm{T}}$ values. At high $V_{\mathrm{A}-\mathrm{C}}$ values, $\xi$ converges asymptotically, with negligible dependence on $V_{\mathrm{C}-\mathrm{T}}$. We obtained $\xi$ values as low as $0.07-0.08$ for gains between $\sim 8 \times 10^{5}-10^{7}$.

Further ion back-flow suppression is possible, by reducing the transfer field $E_{\mathrm{T} 3}$, between GEM3 and the MHSP. This idea was tested in a 4-GEM detector [9], demonstrating back-flow suppression of ions, which is inevitably accompanied with charge transfer suppression of electrons and, thus, with a significant reduction of total gain. However, in the present configuration the total gain is further boosted by the anode-strips multiplication such that values of $\xi \sim 0.03$ for $E_{\mathrm{T} 3}=0.1 \mathrm{kV} / \mathrm{cm}$

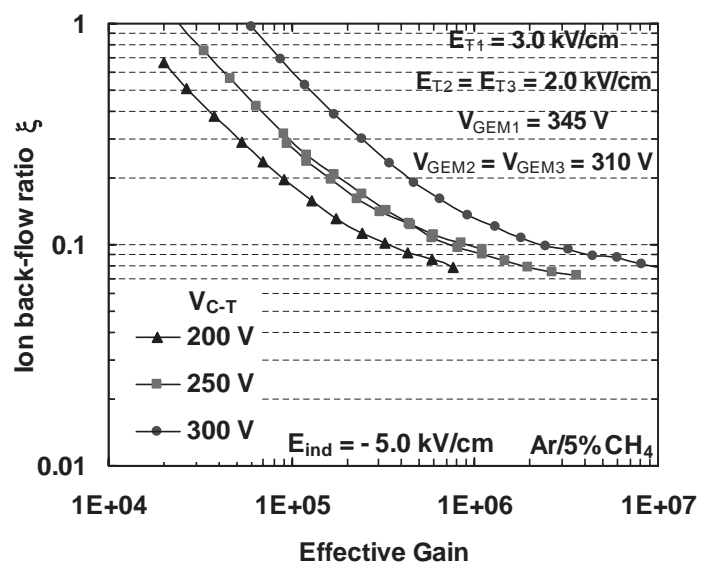

Fig. 10. The avalanche-ion back-flow fraction $\xi$ as function of the effective gain $G_{\text {total }}$ (measured by varying $V_{\mathrm{A}-\mathrm{C}}$ ) for different $V_{\mathrm{C}-\mathrm{T}}$ values, in the multi-GEM/MHSP detector. 


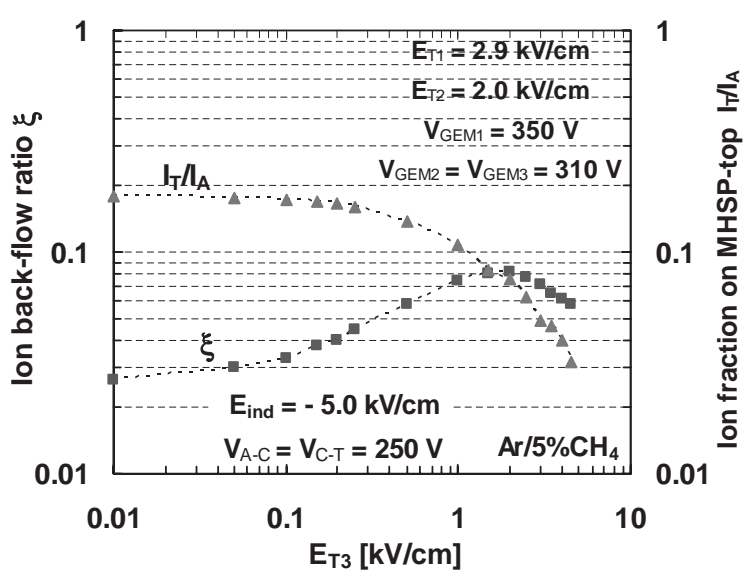

Fig. 11. The avalanche-ion back-flow fraction $\xi$, and the fraction of ions collected on MHSP-top electrode $I_{\mathrm{T}} / I_{\mathrm{A}}$, versus the transfer field, $E_{\mathrm{T} 3}$, in the multi-GEM/MHSP detector. The effective gain $G_{\text {total }}$ is between $\sim 10^{6}$ and $3 \times 10^{6}$.

were obtained at gains in excess of $10^{6}$ as shown in Fig. 11. The fraction of ions collected on MHSP-top, $I_{\mathrm{T}} / I_{\mathrm{A}}$, at this field, is $\sim 0.17$ and $\beta_{\text {top }} \approx 1-\left(I_{\mathrm{T}} / I_{\mathrm{A}}\right) / \varphi=0.26$. Thus, one gets $\beta_{\mathrm{MHSP}}=$ $0.21 \times 0.26 \cong 0.05$, that is to say $95 \%$ of anodestrips ions are collected on the various MHSP electrodes. It is also clear from Fig. 11 that at $E_{\mathrm{T} 3} \sim 2.0 \mathrm{kV} / \mathrm{cm}$, which is a typical value for optimal-gain operation of multi-GEM multipliers, the avalanche-ion back-flow fraction reaches its maximum value, of $\xi \sim 0.08$. $\xi$ decreases at higher fields as more ions are collected on the bottom of GEM3 and at lower fields as more ions are collected on MHSP-top, as evident from the $I_{\mathrm{T}} /$ $I_{\mathrm{A}}$ curve.

Further improvement of the avalanche-ion back-flow fraction $\xi$, was attempted by varying all transfer fields in the multipliers cascade. Fig. 12 depicts $\xi$ as a function of $G_{\text {total }}$ (measured by varying $\left.V_{\mathrm{A}-\mathrm{C}}\right)$ for different $V_{\mathrm{GEM} 2}, V_{\mathrm{GEM} 3}$ and $V_{\mathrm{C}-\mathrm{T}}$ voltages and for $E_{\mathrm{T} 1}=1.0 \mathrm{kV} / \mathrm{cm}$ and $E_{\mathrm{T} 2}=E_{\mathrm{T} 3}=0.25 \mathrm{kV} / \mathrm{cm}$. Under these conditions, the best $\xi$ values are between 0.025 and 0.035 , which is only a small improvement compared to the previous results (Fig. 11). The lowest value of $\xi$, of 0.025 , was recorded with a gain of $\sim 8 \times 10^{5}$, at $V_{\mathrm{GEM} 3}=V_{\mathrm{GEM} 2}=280 \mathrm{~V}$ and $V_{\mathrm{C}-\mathrm{T}}=350 \mathrm{~V}$; such gain is sufficient to operate the photondetector in single-photon-counting mode.

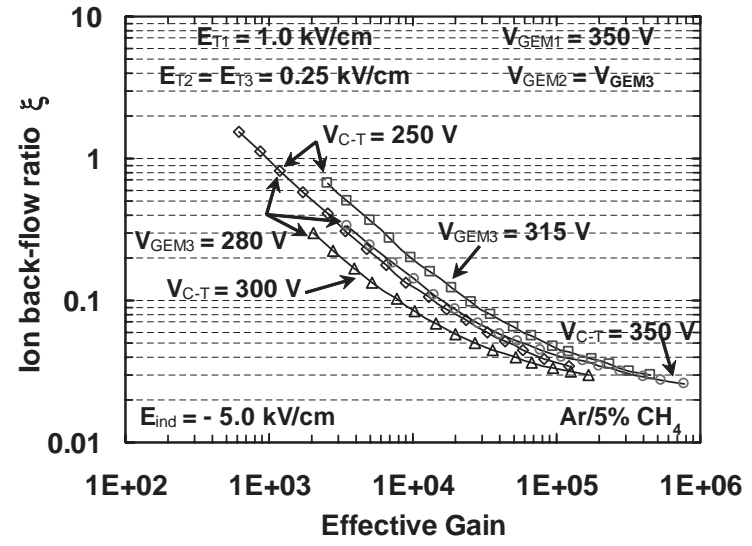

Fig. 12. Avalanche-ion back-flow fraction $\xi$, as a function of the gain $G_{\text {total }}$ (measured by varying $V_{\mathrm{A}-\mathrm{C}}$ ), for different $V_{\mathrm{GEM} 2}, V_{\mathrm{GEM} 3}$ and $V_{\mathrm{C}-\mathrm{T}}$ voltages in the multi-GEM/MHSP detector.

\section{Conclusions}

We have studied the performance of the MHSP multiplier in terms of avalanche-induced ions collection and searched for ways of reducing ion back-flow in cascaded multi-GEM/MHSP electron multipliers.

Studies with a single MHSP coupled to a semitransparent CsI-photocathode were carried out in atmospheric $\mathrm{Ar} / 5 \% \mathrm{CH}_{4}$. The fraction $\varphi$ of backflowing ions is very little influenced by the holes-voltage $V_{\mathrm{C}-\mathrm{T}}$, and the most important parameters are the voltage $V_{\mathrm{A}-\mathrm{C}}$ between anode and cathode strips and the induction field $E_{\text {ind }}$, between the MHSP and the cathode plane underneath it (Fig. 2).

By applying a reversed induction field of -5.0 to $-6.0 \mathrm{kV} / \mathrm{cm}$ rather than a field close to zero, the combined ion-fraction collected on the cathodestrips and on the cathode-plane can be increased by about $30 \%$, and the fraction of back-flowing ions $\varphi$ can be reduced by about a factor 2 . The best results of $\varphi \cong 0.2$, are obtained for the maximum attainable strips-voltage, i.e. around $260 \mathrm{~V}$, and for an induction field in the range of -5.0 to $-6.0 \mathrm{kV} / \mathrm{cm}$.

These results clearly demonstrate a 5-fold reduction in ion back-flow in the MHSP, as compared to a single GEM in which all ions 
propagate upwards through the holes $(\varphi \cong 1.0)$. This improvement is almost independent of the electric field in the MHSP-holes, and is completely independent of the electric fields in the gaps above the MHSP.

Alternatively one can discuss the ion back-flow reduction in terms of the very low ion-transparency $\beta_{\text {MHSP }}$ of the MHSP, found to be in the range of $5-14 \%$. For GEMs the measured ion-transparency is between $30 \%$ and $50 \%$ [8].

The ion-collection proprieties of a photodetector comprising a 3-GEMs cascade followed by an MHSP were systematically investigated; the detector has a reflective CsI-photocathode deposited on top of the first GEM. The efficient collection of avalanche-ions on the cathode-strips and on the cathode-plane below the MHSP, considerably reduces the avalanche-ion back-flow fraction, $\xi$. Additionally, taking advantage of the additional gain-boost occurring at the anode-strips, operation conditions can be found (using a small transfer field in the last transfer gap) that results in about one order of magnitude reduction of the ion back-flow fraction, $\xi$, compared to a 4GEMs photodetector operating at similar gain of about $10^{6}$.

The best result measured for $\xi$ is of $\sim 2.5 \%$, compared to a $20-30 \%$ value obtained in the $4-$ GEMs photodetector [9] (excluding the particular 4-GEMs configuration with the induction region operated in a parallel-plate multiplication mode). It is important to note that the value of $\xi \cong 2.5 \%$ was obtained maintaining high electric field at the photocathode, to assure minimal losses of QE due to photoelectron backscattering on gas molecules.

Nevertheless, our results show that the fraction of avalanche-ion back-flow $\xi$, converges asymptotically with the increase of strips-voltage in the MHSP; similar behaviour was found for GEMs with the increase of the GEM-voltage [6]. It implies that $\xi$ cannot be improved by simply varying the electric fields configuration. A significant reduction in $\xi$ can only be reached by reducing the individual ion-transparencies $\beta$ of each element in the cascade (see Eq. (1) and Eq. (4)), by changing the multiplier's geometric parameters: pitch, hole-diameter and hole-shape, etc. For example, the reduction of the ion- transparency in the MS-side of an MHSP can be done by changing the cathode-strip widths or by using single-conical holes with the smaller diameter on the bottom-side. The latter was similarly suggested and tested for GEMs [8].

The rather low ion back-flow fractions reached in this work have a direct effect of limiting ionfeedback phenomena in photodetectors, in X-ray and neutron detectors with solid converters and gas electron multipliers, and in particle tracking devices. This results in higher attainable multiplication factors. Reduction of ion back-flow is naturally important in time projection chambers (TPCs), where MHSPs could be used as well. In cases where the $2-3 \%$ limit of residual ions is still too high, one can stop the back-drifting ions by applying active ion-gating with a dedicated electrode. It has been recently demonstrated that residual ion levels of the order of $10^{-4}$ could be reached in a 4-GEMs gated detector, though at the expense of a certain dead-time and a counting-rate limit of $\sim 10^{5} \mathrm{~Hz}[9]$.

\section{Acknowledgements}

We would like to thank R. de Oliveira for his help in developing the MHSPs. This work was partially supported by the project CERN/P/FIS/ $43785 / 02$, by the Israel Science Foundation and by the Planning and Budgeting Committee of the Council for Higher Education in Israel. J.M. Maia acknowledges the fellowship provided by the Fundação Calouste Gulbenkian, Lisbon, Portugal. J.F.C.A. Veloso acknowledges support from Fundação para a Ciência e a Tecnologia, Lisbon, Portugal. A. Breskin is the W.P. Reuther Professor of Research in peaceful use of Atomic Energy.

\section{References}

[1] A. Breskin, T. Boutboul, A. Buzulutskov, R. Chechik, G. Garty, E. Shefer, B.K. Singh, Nucl. Instr. and Meth. A 442 (2000) 58.

[2] J. Edmends, D. Miller, F. Barlow, Nucl. Instr. and Meth. A 273 (1988) 145.

[3] F. Sauli, Nucl. Instr. and Meth. A 386 (1997) 531. 
[4] A. Buzulutskov, A. Breskin, G. Garty, R. Chechik, F. Sauli, L. Shekhtmann, Nucl. Instr. and Meth. A 443 (2000) 164 .

[5] D. Mörmann, A. Breskin, R. Chechik, P. Cwetanski, B.K. Singh, Nucl. Instr. and Meth. A 478 (2002) 230.

[6] A. Breskin, A. Buzulutskov, R. Chechik, B.K. Singh, A. Bondar, L. Shekhtman, Nucl. Instr. and Meth. A 478 (2002) 225.

[7] A. Di Mauro, E. Nappi, F. Posa, A. Breskin, A. Buzulutskov, R. Chechik, S.F. Biagi, G. Paic, F. Piuz, Nucl. Instr. and Meth. A 371 (1996) 137.

[8] A. Bondar, A. Buzulutskov, L. Shekhtman, A. Vasiljev, Nucl. Instr. and Meth. A 496 (2003) 325.

[9] D. Mörmann, A. Breskin, R. Chechik, D. Bloch, Nucl. Instr. and Meth. A 516 (2004) 315.

[10] S. Bachmann, A. Bressan, L. Ropelewski, F. Sauli, A. Sharma, D. Mörmann, Nucl. Instr. and Meth. A 438 (1999) 376.
[11] F. Sauli, S. Kappler, L. Ropelewski, IEEE Trans. Nucl. Sci. NS-50 (4) (2003) 803.

[12] M. Balcerzyk, et al., IEEE Trans. Nucl. Sci. NS-50 (4) (2003) 847.

[13] J.F.C.A. Veloso, J.M.F. dos Santos, C.A.N. Conde, Rev. Sci. Instrum. 71 (2000) 2371.

[14] J.F.C.A. Veloso, J.M. Maia, R.E. Morgado, J.M.F. dos Santos, C.A.N. Conde, Rev. Sci. Instrum. 73 (2002) 488.

[15] J.M. Maia, J.F.C. Veloso, R.E. Morgado, J.M.F. dos Santos, C.A.N. Conde, IEEE Trans. Nucl. Sci. NS-49 (3) (2002) 875.

[16] J.M. Maia, J.F.C.A. Veloso, J.M.F. dos Santos, A. Breskin, R. Chechik, D. Mörmann, Nucl. Instr. and Meth. A 504 (2003) 364.

[17] A. Oed, Nucl. Instr. and Meth. A 263 (1988) 351. 